\title{
Design and Implementation of Welding Robot Based on PLC
}

\author{
Litao Wang \\ Shaanxi Polytechnic Institute, Xianyang Shaanxi 712000 China
}

\begin{abstract}
With the development of the revolutionary tide of new technology, our production scale is gradually expanding. Manual production has been unable to meet the requirements of industrial production. In order to improve production efficiency and welding quality, we developed a welding robot. Because it has the characteristics of high production efficiency, stable welding quality and large practical demand. It is developing most rapidly. With the development of PLC technology, we gradually apply it to the development of welding robot. Its advantages of good ductility, low cost and convenient maintenance can bring great value to industrial production.
\end{abstract}

\section{Introduction}

The promotion of science and technology and the requirement of industrial production on science and technology level influence and promote each other. The scale of production required by human society is gradually expanding. Most modern enterprises expand production scale through mass production. The larger the scale of production, the finer the division of labor. Each type of worker performs only a few actions, which are often monotonous and repetitive. These operations can be achieved through mechanical structures [1]. In addition, the efficiency and quality of manual operation are affected by human responsibility, technical level and other factors. It is difficult to guarantee the quality of production completely and produce higher waste products. Some processes cannot be achieved by hand. Manual production cannot meet the requirements of industrial production. Aiming at this phenomenon, a welding robot was invented. They can be used to complete production tasks in harsh environments, improve welding quality and improve production efficiency. How to improve production efficiency and welding quality, the solution of new technology is to develop welding robot [2]. It doesn't worry about the work environment, and it may save physical labor. However, welding robot has very low requirements for operation technology and can shorten the cycle.

The design of robot control system is the most important part of industrial robot [3] design. However, there are a variety of robot control systems [4]. Although the performance of its dedicated controller is very comprehensive, its price is relatively high. Along with the PLC development and the technical consummation, how to select and own control system is the key of this topic. We should combine the economic benefits of the enterprise and use P LC as the controller of the robot to ensure the stability and reliability of the product.

\section{Welding Robot}

Welding robot [5] has been updated for several generations with the development of technology, but the basic components of welding robot are basically unchanged. It mainly has two parts, one is the free movement of the body, the other is driven by some mechanical welding equipment If take arc welding robot as an example, its main components are control cabinet, welding power supply, wire feeder, teaching programmer and welding gun. The control system is mainly divided into two categories: one is non-servo control system [6], which has a general accuracy and low price; Second, servo control system [7] has high precision and high price.

The non-servo control system is a pre-programmed motion track, and then the system controls the motion axis according to the given program for continuous motion. At the end of the motion, it doesn't care if it's in place. Usually, it mainly needs to control a movement according to the feedback signals of various detection switches. This control method is simple and mainly suitable for robot systems with fixed trajectory and simple motion program.

Servo control [8] is a kind of control method commonly used in robots. Sensors are used to detect movement and collect information such as position and speed of components. Then, it sends the detection results to the corresponding control system, and finally the system continuously corrects its motion trajectory according to the feedback data. And then finally it detects if the motion is in place. This kind of method is more complex, but its positioning accuracy is higher and its application is more extensive.

Figure 1 shows the servo control system. The servo control system has a very high control precision, the system USES a variety of sensors to continuously track the precise position of the joint and other parameters, the detected data feedback to the controller to form a closedloop servo system. However, under the control of the 
server, the position and speed of each joint are controlled by the corresponding program. The internal program debugging is more flexible, the system can quickly complete complex operations. At present, servo control system is basically used in most high-performance robots.

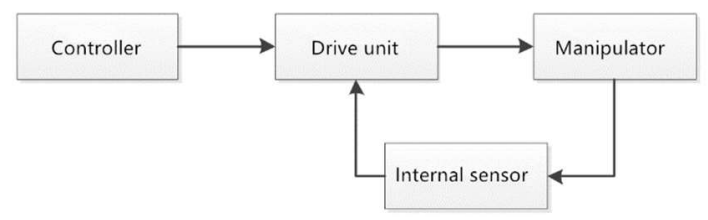

Figure 1. Closed-loop servo system

\section{PLC control system}

The control system can act as the brain of the robot and determine the movement of the robot. Robot control system has many forms, and its advantages and disadvantages are different. Robots generally need to have strong computing power. PLC is used as the controller to connect the wire in a simple and easy programming way, which can directly use special instructions to complete the multi-axis control of the robot. One of the characteristics of PLC is PLC as a controller without complicated circuit design. It assigns values directly to the terminals and then connects them for control. The robot controller usually takes the microprocessor as the center, and USES tools such as motion trajectory design, programming software and control algorithm to achieve accurate motion control. Usually, in the control process, the acceleration, specific speed, position and other parameters of the detection element are fed back in time according to the results, and finally the relevant instructions are adjusted to achieve this.

The classical servo control system has many forms. According to the analysis of the control theory, the system can be divided into five parts: the controlled object, the controller, the link to perform the work, the comparison and the test. The parameters controlled by the system are carried out through the controlled object, usually including velocity, displacement and torque. The execution work link sends out a series of signals according to the controller, converts the corresponding control instructions into specific actual mechanical movements according to the requirements, and finally drives the actuator to work. Usually the actuator is usually a motor or manipulator, etc. The controller control loop is PID. It is necessary to efficiently convert the output deviation signal and control each actuator according to the requirements of the instruction, so as to complete the corresponding steps. The comparison link is to detect the data and feed it back to the system, then compare it with the system signal, and calculate the error according to the comparison data. It is necessary to continuously measure the output data between the systems, and then change the required data into a device in the comparison link. At this time, the link is usually the sensor and the transformation loop.

Robot operations are generally reproduced through real-time programming, as shown in figure 2. According to the position of the robot and the surrounding environment, the operation flow and the corresponding movement path of a certain robot are set. According to its functions, it is divided into four parts: basic functions and parameters Number setting, teaching and other functions.

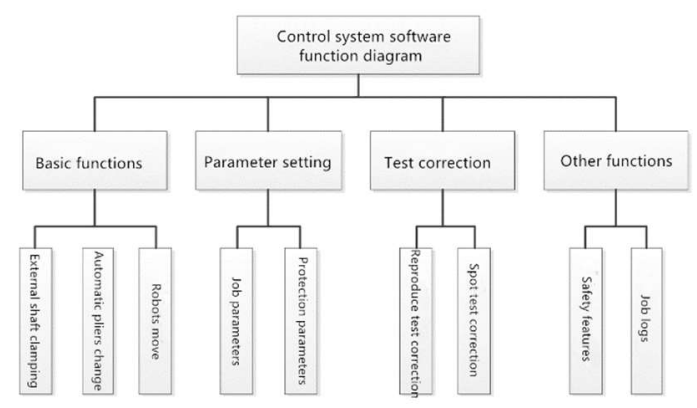

Figure 2. Control system function diagram of welding robot

The parameter setting here includes operation parameters and protection parameters. Because robot programming requires real-time performance, real-time detection of safety signals is required. PLC has many interfaces and modules, generally used in industrial control field of control system. PLC built-in many programming languages, according to the specific task can be faster to write a program. These programs can be debugged on the computer, but also can be stored directly in the PLC internal, improve the executability and extensibility, and then easier to achieve process control. PLC can also be used as industrial control computer, its hardware structure and microcomputer similar.

\section{Robot control}

The driving device of the robot is an electric motor, which then pushes the motion between the joints to be executed. Therefore, the robot needs to have unique requirements for the motor, including low moment of inertia and high starting torque. The motor of end-effector is required to be as small as possible in order to reduce its moment of inertia and reduce the difficulty of the system. To ensure high speed response and reliability is the key factor of motor selection. The motor control of joint drive is to set the trajectory and determine the key position points, as shown in FIG. 3. The steps are as follows:

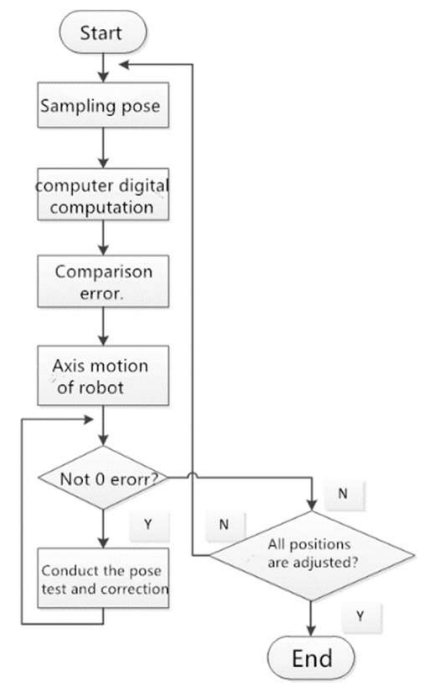

Figure 3. Control flow chart 
Firstly, the sensor signal at the joint position can judge the position of the end-effector, and the sensor signal can be transmitted to the main computer, and then transformed, and finally the pose data can be obtained.

Secondly, the main computer can solve inverse kinematics, solve the vector of each joint, and then store it.

Once again, the positions of the joints and the actual positions are compared, the deviation signals are obtained, and the motor is automatically accelerated and decelerated according to the deviation size, so as to ensure that the deviation is small.

As the robot moves, various parameters are generated and recorded, which can provide the basis for adjusting the motion at any time.

Then, when the deviation signal is close to zero, the robot will stop. At this point, it means that the robot has reached the position of instruction and will prepare and send out the next pose information. The robot will continue to repeat the above steps.

\section{Conclusion}

Under the revolutionary tide of new technology, many foreign developed countries apply robot technology in large quantities. Welding robot is an important part of industrial robot. Welding is a high precision but heavy labor, so the manufacture of welding robot can liberate the labor force, promote the development of industrial robots. It has been widely used in more and more enterprises.

\section{References}

1. Chen haichu, Cao Quan, Xiong Genliang, Yang Piao, Sun Junlong. Design and implementation of programmable welding robot teaching system [J]. Hot processing technology, 2016, 45 (19): 179-182.

2. Li Zhiqiang, Guo Guangling, Fan Ding, Shi Yu. Arc welding robot with $\mathrm{NC}$ welding displacement machine teaching system $[\mathrm{J}]$. The design and implementation of manufacturing technology and machine tool, 2004 (8): 61-61.

3. He Xingxing, Zhang Yixin. Use SOLIDWORKS for welding robot design and simulation $[\mathrm{J}]$. Journal of $\mathrm{CAD} / \mathrm{CAM}$ and manufacturing information, 2014 (12): 47-49.

4. Yuan Junfeng, Yi Guochun, Xu Suexin, Geng Yunxiang, Zhang Hongjian. Design and implementation of automatic welding robot workstations for security doors in housing [J]. Automation of manufacturing industry, 2014, 36 (21): 146-148.

5. Liu Shengpeng, Ye Hua, Wang Dan. Design and implementation of safety controller for industrial welding robot $[\mathrm{J}]$. Industrial control computer, 2009, 22 (10): 13-14.

6. Huang Shouning, Jiang Chaoning, Dai Jianshu. Design and implementation of welding robot controller based on S3C2440 [J]. Manufacturing automation, 2009, 31 (09): 169-171.

7. Zhang Yang, Wang Xuanyin, Liu Songguo. Design and implementation of embedded teaching system for welding robot $[\mathrm{J}]$. Modular machine tool and automation processing technology, 2007 (03): 1-3+7.

8. Jin Yunxiang, Jiang Lipei, Zou Yong, Chen Haiyang, Sun Yaling, Xu Feng. Design and implementation of interactive control system for pipeline welding robot $[\mathrm{J}]$. Journal of Beijing university of petroleum and chemical technology, 2006 (01): 24-27. 